\title{
Does the stability above the upper boundary have any effect on the orographic rainfall?
}

\author{
Somenath Dutta ${ }^{1}$
}

Received: 24 March 2016/Accepted: 26 March 2016/Published online: 7 April 2016

(C) Springer International Publishing Switzerland 2016

\begin{abstract}
An attempt has been made to examine whether the stability condition of the layer, above the upper boundary of a computational domain, has any effect on the magnitude and spatial pattern of rainfall intensity (RFI), as diagnosed by dynamical model of orographic rainfall. For this purpose a three dimensional mesoscale dynamical model for orographic rainfall has been used. In this model the vertical computational domain has been capped by layer (here after called top layer) with a constant background wind and stability. With this slight modification the model is being run with different values of stability of the top layer for a given vertical profile of wind and temperature at a station, far upstream of the orographic barrier. Preliminary results show that given the profile of a 3-D meso scale mountain, the vertical profile of basic flow across it, the magnitude and spatial distribution (1-D and 2-D) of computed orographic rainfall intensity hardly have any bearing with top layer stability. Interestingly, in some cases it has been found that the effect of imposition of a top layer is to reduce the magnitude of peak computed RFI just behind the barrier and thus underestimation of RFI.
\end{abstract}

Keywords Orographic rainfall · Top layer stability

\section{Introduction}

Dynamical parameterization of orographic rainfall near a meso scale orographic barrier has been a challenge to mesoscale numerical weather prediction. To meet it, in

Somenath Dutta

dutta.drsomenath@gmail.com

India Meteorological Department, Pune, India earlier studies separate dynamical model has been proposed to diagnose orographic rainfall. Such parameterization may be found in the studies by Murray (1948), Sawyar (1956), Sarker (1966, 1967), Collier (1975), Rhea (1978), Sarker et al. (1978), Sinclair (1994), Smith and Barstad (2004) and Dutta (2007).

The above studies may broadly be divided into two categories. In one category a constant wind and stability of the basic flow throughout the model vertical domain has been assumed, for example, Smith and Barstad (2004) etc. In this category, generally radiation upper boundary condition is imposed. In the other category realistic vertical profiles of wind and temperature at a station far upstream of the barrier are taken, for example, Sawyar (1956), Sarker (1966, 1967), Sarker et al. (1978), Dutta (2007). Fixing a suitable upper boundary condition in the later category study has posed a great diffculty, because of the fact that beyond a certain level wind and temperature data at a station far upstream of the barrier are not available. Due to this, most of the studies in this category assume a top layer with constant wind and moist neutrally stable.

At this juncture a question naturally arises, whether the stability condition of the top layer has any effect on the magnitude and spatial pattern of computed RFI. For two-dimensional studies, Corby and Sawyar (1958), Palm and Foldvic (1960) and Sawyar (1960) had shown that the wind and stability conditions far aloft have hardly any effect on low-level flow. For three-dimensional flow hardly any study has addressed this problem.

Objective of this study is to investigate the effect of top layer stability condition on the magnitude and spatial pattern of computed orographic RFI, with special emphasis on Indian cases. 


\section{Data}

Lonavla and Khandala are two stations located to the windward side of Mumbai-Pune section of the WG. Daily rainfall data of these two stations, required for validation of result, have been obtained from the archive of India Meteorological Department. RS/RW data of station located at far upstream of the WG (Santacruz), required to initialize Dutta (2007) model have been obtained from the archive of India Meteorological Department. The above model also be initialized by the vertical profiles of wind, temperature, geopotential and pressure of gridpoint nearest to above far upstream station, prepared using large scale/regional scale/ $\operatorname{meso}(\alpha)$ scale model analyzed/forecast values of above field. We have used ERA-40data for such case.

\section{Methodology}

To examine the effect of top layer stability on the magnitude and spatial pattern of computed orographic RFI, a 3-D meso-scale linear dynamical model for orographic rainfall (Dutta 2007) has been used.

\section{Brief about the dynamical model used}

In this model there are two parts, viz., a dynamical part and a thermo dynamical part. In the dynamical part the model computes the vertical velocity $\left(w^{\prime}\right)$ due to perturbation induced by the orographic barrier and in the thermo dynamical part it computes the rainfall intensity, using the perturbation vertical velocity $\left(w^{\prime}\right)$, basic state density $(\rho)$ and basic state saturation mixing ratio $(q)$.

The model considers a saturated adiabatic, non-rotational, Boussinesq and laminar basic westerly flow under a steady-state condition. Under the above assumptions the linearised governing equations are given below:

$$
\begin{aligned}
& U\left(\frac{\partial u^{\prime}}{\partial x}\right)+w^{\prime}\left(\frac{d U}{d z}\right)=-\left(\frac{1}{\rho_{0}}\right)\left(\frac{\partial p^{\prime}}{\partial x}\right) \\
& U\left(\frac{\partial v^{\prime}}{\partial x}\right)=-\left(\frac{1}{\rho_{0}}\right)\left(\frac{\partial p^{\prime}}{\partial y}\right) \\
& U\left(\frac{\partial w^{\prime}}{\partial x}\right)=-\left(\frac{1}{\rho_{0}}\right)\left(\frac{\partial p^{\prime}}{\partial z}\right)-g\left(\frac{\rho^{\prime}}{\bar{\rho}}\right) \\
& \left(\frac{\partial u^{\prime}}{\partial x}\right)+\left(\frac{\partial v^{\prime}}{\partial y}\right)+\left(\frac{\partial w^{\prime}}{\partial z}\right)=0 \\
& U\left(\frac{\partial \theta_{e}^{\prime}}{\partial x}\right)+w^{\prime}\left(\frac{d \bar{\theta}_{e}}{d z}\right)=0
\end{aligned}
$$

The above equations are subjected to 2-D Fourier transformation. The Fourier transformed equations, after some algebraic simplification, are reduced to the following vertical structure equation:

$$
\begin{aligned}
& \frac{\partial^{2} \tilde{w}}{\partial z^{2}}+\frac{1}{\rho_{0}} \frac{d \rho_{0}}{d z} \frac{\partial \tilde{w}}{\partial z} \\
& \quad+\left(\frac{N_{m}^{2}\left(k^{2}+l^{2}\right)}{(U k)^{2}}-\frac{1}{U} \frac{d^{2} U}{d z^{2}}-\frac{1}{\rho_{0}} \frac{d \rho_{0}}{d z} \frac{1}{U} \frac{d U}{d z}-\left(k^{2}+l^{2}\right)\right) \tilde{w}=0
\end{aligned}
$$

Using the substitution,

$\tilde{w}(k, l, z)=\sqrt{\frac{\rho_{0}(0)}{\rho_{0}(z)}} \tilde{w}_{1}(k, l, z)$

Eq. (6) is further simplified to:

$$
\begin{aligned}
& \frac{\partial^{2} \hat{w}_{1}}{\partial z^{2}}+\left(\frac{N_{m}^{2}\left(k^{2}+l^{2}\right)}{(U k)^{2}}-\frac{1}{U} \frac{d^{2} U}{d z^{2}}-\frac{1}{\rho_{0}} \frac{d \rho_{0}}{d z} \frac{1}{U} \frac{d U}{d z}\right. \\
& \left.-\left(k^{2}+l^{2}\right)+\frac{1}{4 \rho_{0}^{2}}\left(\frac{d \rho_{0}}{d z}\right)^{2}-\frac{1}{2 \rho_{0}} \frac{d^{2} \rho_{0}}{d z^{2}}\right) \hat{w}_{1}=0
\end{aligned}
$$

Considering the second order of smallness of the last two terms within parenthesis, the above equation further simplified to

$\frac{\partial^{2} \tilde{w}_{1}}{\partial z^{2}}+\left(f(k, l, z)-\left(k^{2}+l^{2}\right)\right) \tilde{w}_{1}=0$.

where, $f(k, l, z)=\left(\frac{N_{m}^{2}\left(k^{2}+l^{2}\right)}{(U k)^{2}}-\frac{1}{U} \frac{d^{2} U}{d z^{2}}-\frac{1}{\rho_{0}} \frac{d \rho_{0}}{d z} \frac{1}{U} \frac{d U}{d z}\right)$.

Here, $\tilde{w}(k, l, z)$ is the 2-D Fourier transformation of perturbation vertical velocity

$w^{\prime}(x, y, z), N_{m}^{2}=\frac{g}{\beta \overline{\theta_{e}}\left(1+q_{s}\right)} \frac{d \overline{\theta_{e}}}{d z}$

and

$\beta=1-\frac{L q_{s}}{C_{p} \bar{T}}\left(\frac{\varepsilon+\gamma q_{s}}{\varepsilon+q_{s}}\right)$

here, $\gamma$ is the ratio between two specific heats of gas, $\varepsilon$ is the ratio between dry and moist gas constants and $q_{s}$ is the basic state saturated mixing ratio of water vapour.

In the above model, Eq. (8) was solved quasi-numerically using following boundary conditions:

(i) At the lower boundary, i.e., at the surface $(z=0)$ airflow is tangential to the ground surface.

(ii) Above the upper boundary basic flow was assumed to be neutrally stratified with a constant velocity. Thus in the above model it was assumed that above the upper boundary, $f(k, l, z) \approx 0$, which implies that at and above the upper boundary,

$\tilde{w}_{1}(k, l, z) \propto e^{-\kappa z}$.

Since the pressure, vertical velocity are continuous functions of $\mathrm{z}$, therefore, $\tilde{w}_{1}, \frac{\partial \tilde{w}_{1}}{\partial z}$ are also continuous functions of z. Hence, above the upper boundary, 
$\frac{\partial \tilde{w}_{1}}{\partial z}=-\kappa \tilde{w}_{1}$

Equations (11) and (12) provided upper boundary conditions in the above model.

(iii). Lateral boundary condition is periodic.

\section{Modification in the dynamical model for the present study}

In the present study we modify the upper boundary condition by imposing a stable top layer with finite depth and constant wind above the actual vertical computational domain. Hence for the present study $\tilde{w}_{1}(k, l, z)$ satisfies Eq. (8) in the actual computational domain and satisfies the equation $\frac{\partial^{2} \tilde{w}_{1}}{\partial z^{2}}+\lambda^{2} \tilde{w}_{1}=0$ in the top layer, where, $\lambda^{2}=\frac{\kappa^{2}}{k^{2}}\left(\frac{N_{m}^{2}}{U_{c}^{2}}-k^{2}\right), U_{c}$ being constant wind in top layer. Numerical experiment has been carried out with different values of $N_{m}$ in the top layer. To solve above equations numerically, we consider an arbitrary function $\psi(k, l, z)$, which satisfies following equations:

$\frac{\partial^{2} \psi}{\partial z^{2}}+\left\{f(k, l, z)-\kappa^{2}\right\} \psi=0$

in the actual computational vertical domain,

$\frac{\partial^{2} \psi}{\partial z^{2}}+\lambda^{2} \psi=0$

in the top layer and

$\psi(k, l, z)=1.0$

at the top of top layer. This modification is schematically shown in Fig. 1. Following Dutta (2005), it can be shown that $\tilde{w}_{1}, \psi$ are linearly dependent, so that former may be expressed as a multiple of later one.

\section{Numerical solution}

The above system of Eqs. (13, 14 and 15) has been solved numerically for the atmospheric layer consisting of the top layer and the actual vertical computational domain. To solve above system, 'N' equally spaced (=d) discrete vertical grids have been inserted in the actual vertical domain and ' $\mathrm{M}$ ' grids in the top layer. The numerical solution for $\psi$, for a given wave number vector $(k, l)$, at different vertical grid points are given below:

$\psi_{m+n+1}=1.0$

For $\lambda^{2}>0.0$ $\psi_{m+n}=1.0+i d \lambda-\frac{d^{2} \lambda^{2}}{2}$

$\psi_{j-1}=-\psi_{j+1}+\left[2-\lambda^{2} d^{2}\right] \psi_{j} \quad$ for

$j=M+N, M+N-1, \ldots, N+2$.

For, $\lambda^{2}<0.0$, assume, $\mu^{2}=-\lambda^{2}$,

$\psi_{m+n}=1.0+\mu d+\frac{d^{2} \mu^{2}}{2}$

$\psi_{j-1}=-\psi_{j+1}+\left[2+\mu^{2} d^{2}\right] \psi_{j}$ for

$j=M+N, M+N-1, \ldots, N+2$

Also, the numerical solution for $\psi(k, l, z)$, at ' $\mathrm{N}$ ' vertical grid points in the actual computational vertical domain are given as follows:

$\psi_{j-1}=-\psi_{j+1}+\left[2.0-d^{2}\left\{f(k, l, z)-\kappa^{2}\right\}\right] \psi_{j}$

for $\mathrm{j}=\mathrm{N}+1, \mathrm{~N}, \ldots, 2$.

After finding out $\psi$ at each vertical grid for every wave number, following Dutta (2007), $\tilde{w}(k, l, z)$ is given by,

$\tilde{w}(k, l, z)=\sqrt{\frac{\rho_{0}(0)}{\rho_{0}(z)}} i k U(0) \tilde{h}(k, l) \frac{\psi(k, l, z)}{\psi(k, l, 0)}$

Then perturbation vertical velocity, $w^{\prime}(x, y, z)$ is obtained by applying numerical $2 \mathrm{D}$ inverse Fourier transform for the wave numbers ranging $l$ from $-20 \delta l$ to $20 \delta l$ and $k$ from $4 \delta k$ to $20 \delta k$, where $\delta k=\delta l=\frac{2 \pi}{4 L_{\max }}$ and $L_{\max }=150 \mathrm{~km}$ is the maximum horizontal scale of the disturbance. Rainfall intensity (RFI) in $\mathrm{mm} / \mathrm{h}$ are computed, incorporating down drift effect, at every grid point in the domain of interest following Dutta (2007).

In the present study the orographic barrier has been approximated by 3-D elliptical barrier with analytical expression, $h(x, y)=\frac{H}{1+\left(\frac{x}{a}\right)^{2}+\left(\frac{y}{b}\right)^{2}}$. Here, ' $H$ ' is the maximum height of the barrier at the centre of the domain, ' $a$ ' is the half width along the minor ridge and ' $b$ ' is that along the major ridge of the above elliptical barrier. In this study $\mathrm{x}$-axis is always along the minor ridge, $\mathrm{y}$-axis along the major ridge and $\mathrm{z}$-axis pointing vertically upwards, irrespective of the barrier.

\section{Results and discussions}

The above model has been run using the RS/RW data of Santacruz, a station far upstream of the Western Ghat along the West coast of India. The region of the present study is a square of side $600 \mathrm{~km}$ with Vadgaon $\left(73^{\circ} 30^{\prime} \mathrm{E}, 18^{\circ} 45^{\prime} \mathrm{N}\right)$ at 


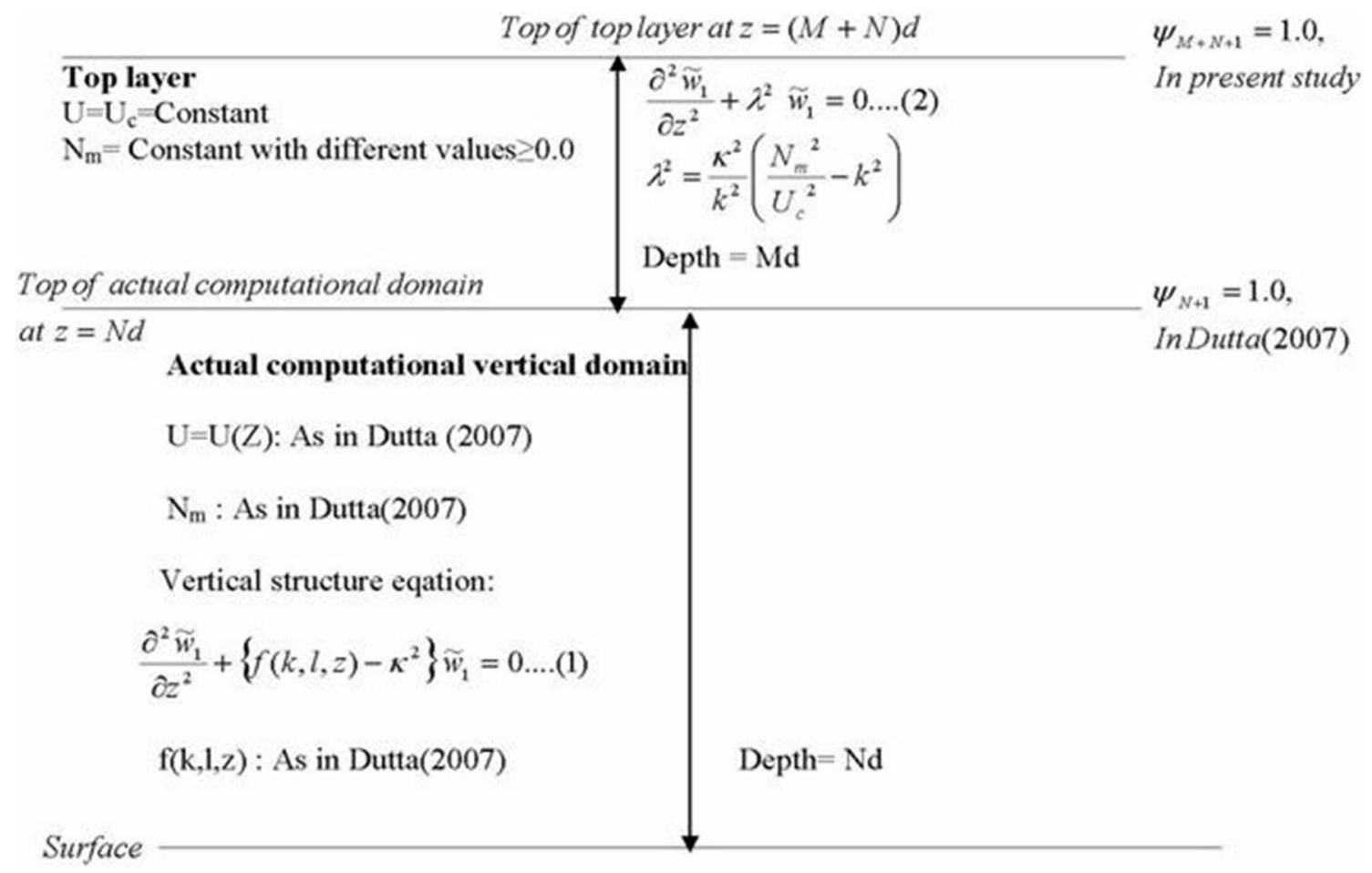

Fig. 1 Schematic diagram of modification of the dynamical model, Dutta (2007)

its center. Vadgaon is a place in the WG region, within the above square, with maximum height. In that region the actual topography has been approximated with an elliptical barrier the contour of which is analytically expressed as given earlier. Numerical experiments have been conducted for a strongly stratified top layer with $N_{m}=1.0 e-04,1.0 e-03,1.0 e-02$, for a weakly stratified top layer with $N_{m}=1.0 e-10,1.0 e-09,1.0 e-08$, $1.0 e-07,1.0 e-06,1.0 e-05$, and also for a neutral top layer. Experiments have also been conducted using no artificial top layer with any stratification as in Dutta (2007). In the above experiments we have taken $4 \mathrm{~km}$ to be the depth of top layer and $U=1 \mathrm{~m} / \mathrm{s}$ through out the top layer. Similar experiments have been conducted for a number of cases using RS/RW data of Santacruz, Of course the realistic orographic barrier has been approximated by a threedimensional elliptical barrier with analytical expression given above. The results obtained are almost similar. Thus for brevity results of only two representative cases (for $3 \mathrm{rd}$ July 2001 and 4th July 2001) for $N_{m}=1.0 e-2,1.0 e-$ $3,1.0 e-8,1.0 e-10,0.0$ and also for the case using no artificial top layer, are presented here for discussions.

\section{Case-1: orographic precipitation due to forced ascent over the Western-Ghat on 3-7-01}

For this case the RS/RW data of Santacruz for the date 3-701 and the observed daily rainfall data of Lonavla for the date 3-7-01 have been utilized. The vertical profiles of wind and temperature are given in Fig. 2(a). In this case the depth of the computational domain is $8.5 \mathrm{~km}$ and that of top layer has been taken as $4 \mathrm{~km}$. Both the above domains have been divided into 34 and 16 discrete layers, respectively, with depth $0.25 \mathrm{~km}$. The distributions of RFI (mm/ h), computed using the above mentioned modified model, in the central plane for strong stable stratification $\left(N_{m}=\right.$ $1.0 e-2,1.0 e-3)$, for weak stable stratification $\left(N_{m}=\right.$ $1.0 e-8,1.0 e-10)$, for neutral stratification and also for no artificial top layer have been given in Fig. $2 b-g$.

From the above figures it can be seen that pattern in the distributions of RFI $(\mathrm{mm} / \mathrm{h})$ in the central plane are similar whether a top layer is capped or not and also irrespective of top layer stability. From these figs it also appears that if no artificial top layer is capped, then the computed peak RFI is $5.52 \mathrm{~mm} / \mathrm{h}$ located at $5 \mathrm{~km}$ upstream of the peak of the barrier and if an artificial top layer, with any stable/neutral stratification, is imposed then it is about $3.5 \mathrm{~mm} / \mathrm{h}$ located at the same place and the observed one is $6.3 \mathrm{~mm} / \mathrm{h}$ located at Lonavla, about $5 \mathrm{~km}$ upstream of the peak. Thus the computed RFI without using any artificial top layer appears to be closer to observed one and imposing a top layer is to reduce the magnitude of peak computed RFI and thus underestimation of RFI.Contours of RFI in the 2-dimensional plane with different values of top layer stability are shown in Fig. 2h-m. In all these figs, as in Dutta (2007), four areas with maximum RFI can be seen, with one 
Fig. 2 a Vertical profile of wind $(\mathrm{U})$ and temperature $(\mathrm{T})$ of basic flow for case 1 .

b Distribution of RFI in the central plane along $\mathrm{y}=0$ when $\mathrm{Nm}=1.0 \mathrm{e}-02$ for case 1 . c Distribution of RFI in the central plane along $\mathrm{y}=0$ when $\mathrm{Nm}=1.0 \mathrm{e}-03$ for case 1 . d Distribution of RFI in the central plane along $\mathrm{y}=0$ when $\mathrm{Nm}=1.0 \mathrm{e}-08$ for case 1 . e Distribution of RFI in the central plane along $\mathrm{y}=0$ when $\mathrm{Nm}=1.0 \mathrm{e}-10$ for case 1 . f Distribution of RFI in the central plane along $\mathrm{y}=0$ when $\mathrm{Nm}=0$ for case 1 .

g Distribution of RFI in the central plane along $\mathrm{y}=0$ without any top layer for case 1 . h Contour of RFI for $\mathrm{Nm}=1 \mathrm{e}$ 2 for case 1 . i Contour of RFI for $\mathrm{Nm}=1 \mathrm{e}-3$ for case 1 .

j Contour of RFI Nm $=1.0 \mathrm{e}-$ 08/s for case- 1 . k Contour of RFI for $\mathrm{Nm}=1 \mathrm{e}-10$ for 3.7 .01 for case 1. 1 Contour of RFI for $\mathrm{Nm}=0$ for case 1 . $\mathbf{m}$ Contour of RFI for case 1 without any top layer

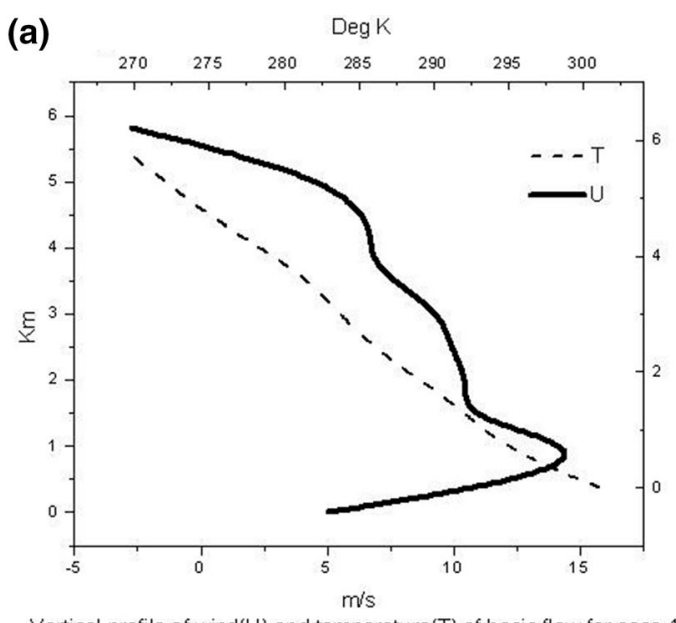

Vertical profile of wind(U) and temperature(T) of basic flow for case 1.
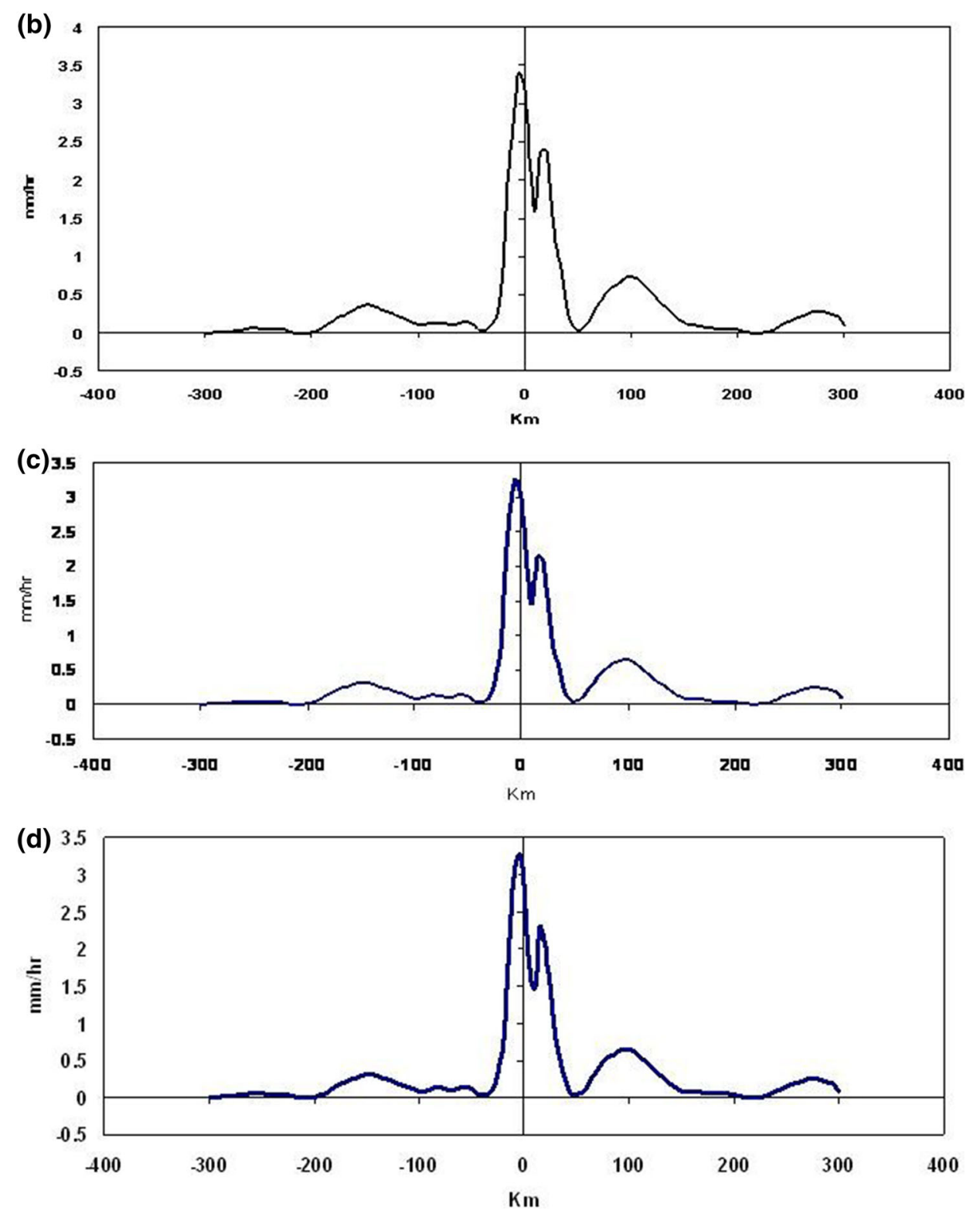

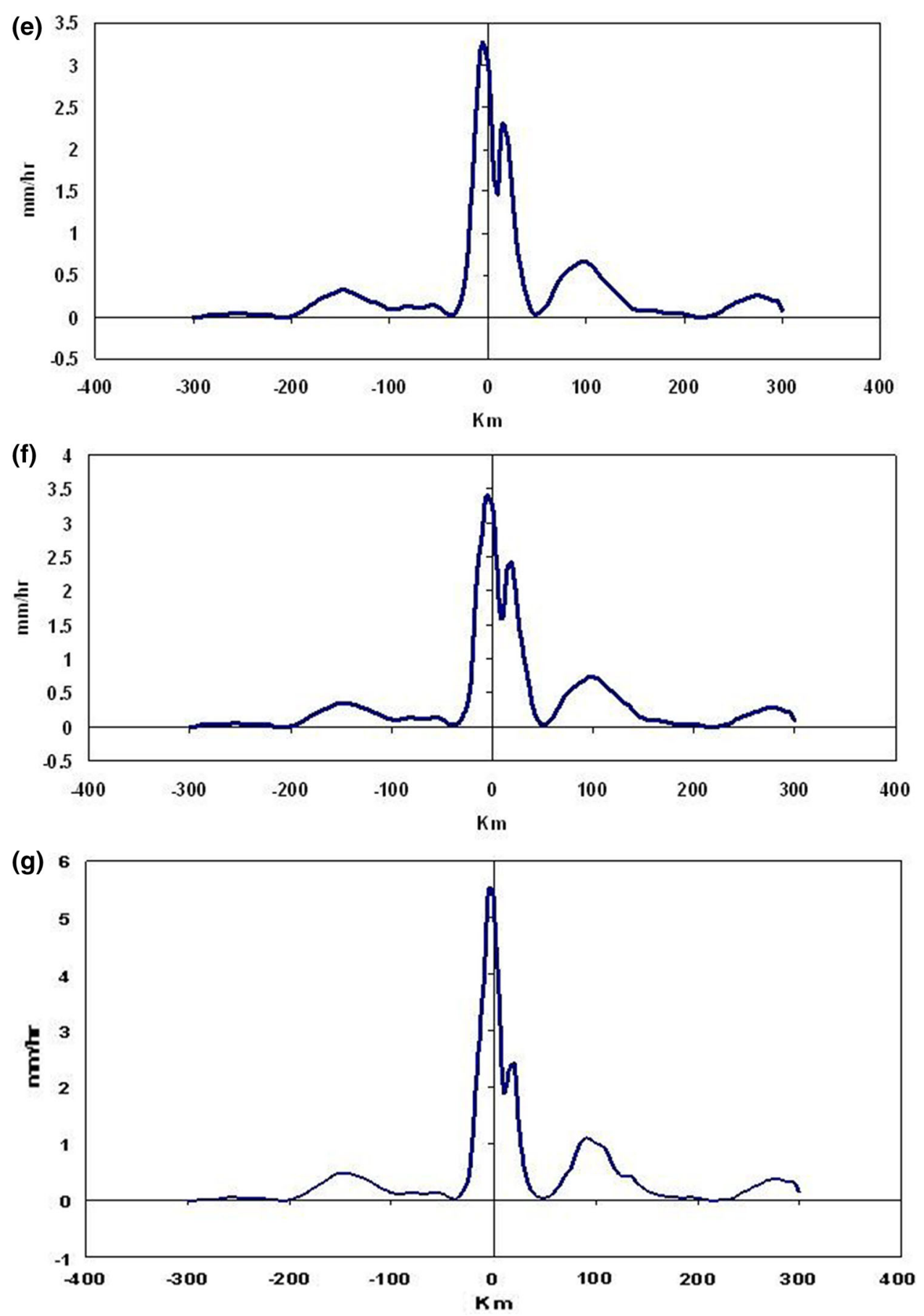

Fig. 2 continued

primary maxima and three secondary maxima. The primary maxima is located in the central plane windward side of the barrier, one secondary maxima is located in the central plane to the leeward side and another two located symmetrically near the flanks of the barrier on lee side. These figs also show that stability of the top layer has hardly any effect on the spatial (2-D) distribution of computed orographic rainfall.

\section{Case-2:orographic precipitation due to forced ascent over the Western-Ghat on 4-7-01}

For this case the RS/RW data of Santacruz for the date 4-701 and the observed daily rainfall data of Lonavla for the date 4-7-01 have been utilized. The vertical profiles of wind and temperature are given in Fig. 3a. Like case-1, in this case also, the depth of the computational domain is 

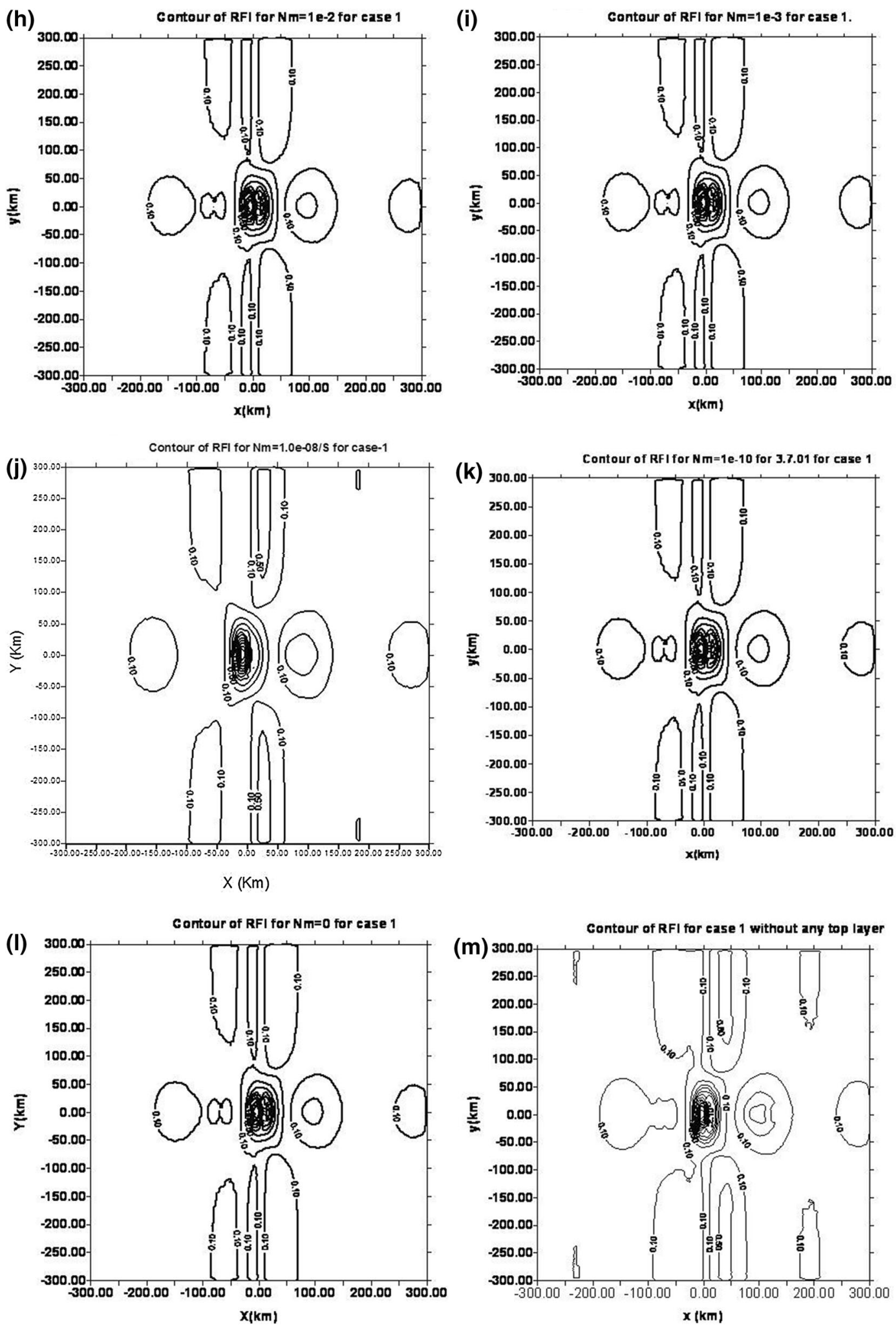

Fig. 2 continued 
Fig. 3 a Vertical profile of wind $(\mathrm{U})$ and temperature $(\mathrm{T})$ of basic flow for case 2. b. RFI in the central plane for

$\mathrm{Nm}=1.0 \mathrm{e}-2$ for case 2 . $\mathrm{c}$ RFI in the central plane for

$\mathrm{Nm}=1.0 \mathrm{e}-3$ for case 2 . $\mathbf{d}$ RFI in the central plane for

$\mathrm{Nm}=1.0 \mathrm{e}-8$ for case $2 . \mathrm{e} \mathrm{RFI}$ in the central plane for case 2 when $\mathrm{Nm}=1.0 \mathrm{e}-10$. $\mathbf{f}$ RFI in the central plane for $\mathrm{Nm}=0$ for case 2. $\mathbf{g}$ RFI in the central plane for case 2 without a top layer. $\mathbf{h}$ Contour of RFI for $\mathrm{Nm}=1.0 \mathrm{e}-2$ for case 2 .

i Contour of RFI for

$\mathrm{Nm}=1.0 \mathrm{e}-3$ for case 2 .

j Contour of RFI for

$\mathrm{Nm}=1.0 \mathrm{e}-8$ for case 2 .

k Contour of RFI $(\mathrm{mm} / \mathrm{h})$ for

$\mathrm{Nm}=1.0 \mathrm{e}-10$ for case 2 .

1 Contour of RFI for $\mathrm{Nm}=0$

for case 2. $\mathbf{m}$ Contour of RFI

$(\mathrm{mm} / \mathrm{h})$ for no top layer for case 2 (a)

Deg K

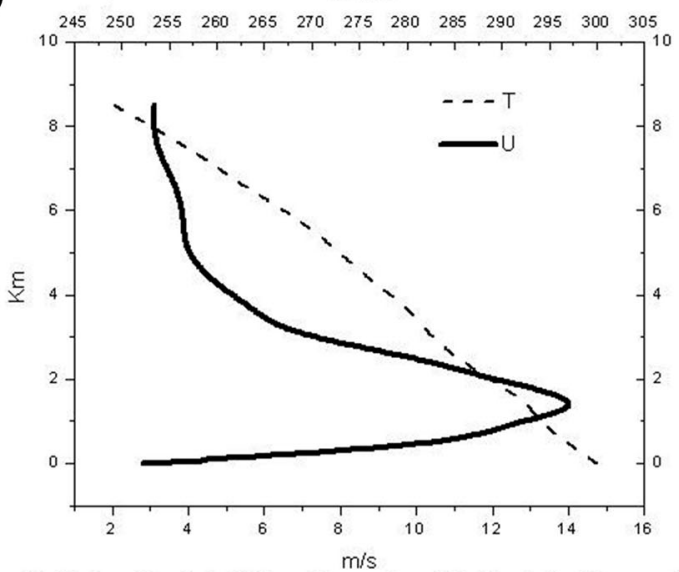

Vertical profile of wind $(U)$ and temperature(T) of basic flow for case 2 .
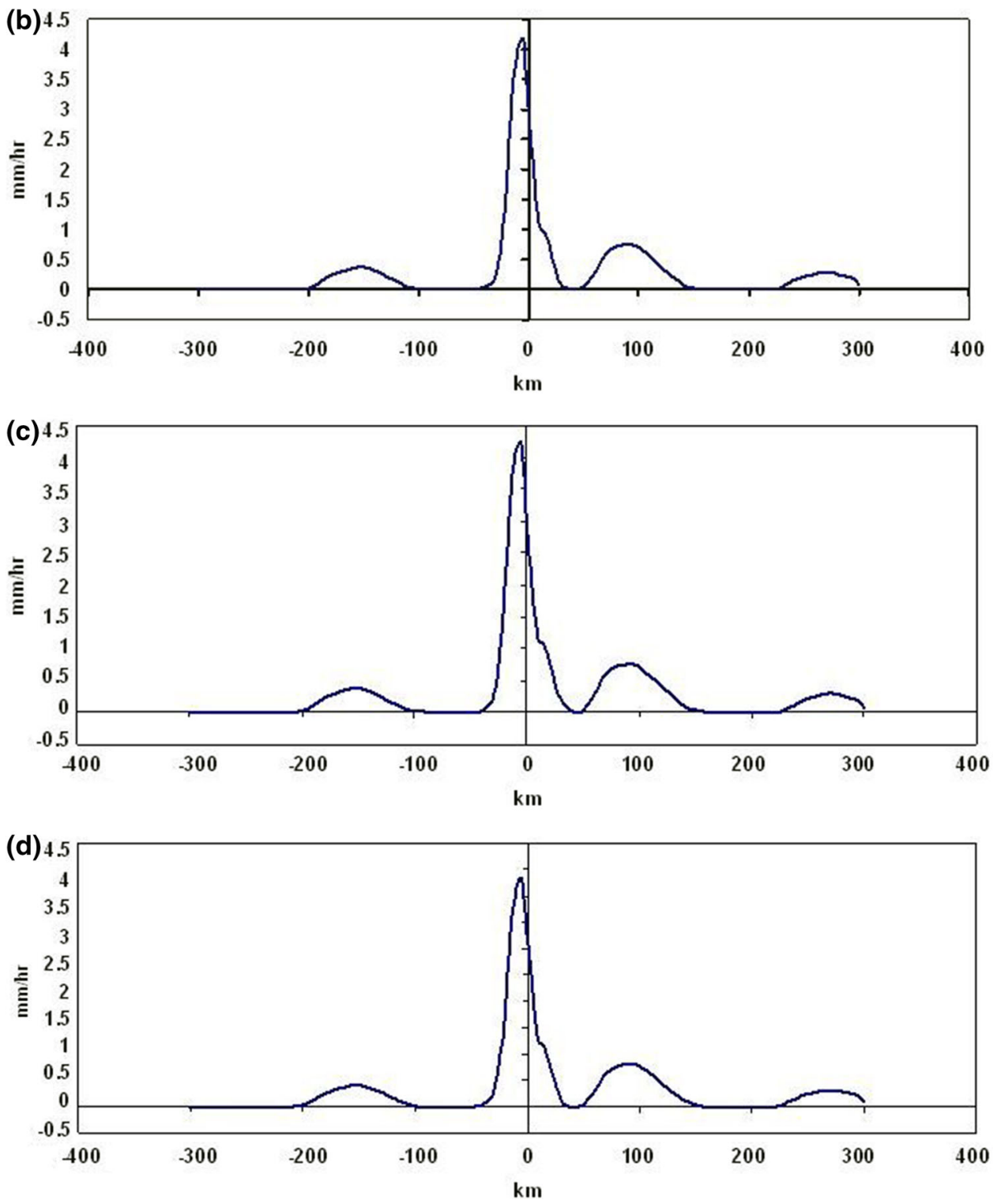

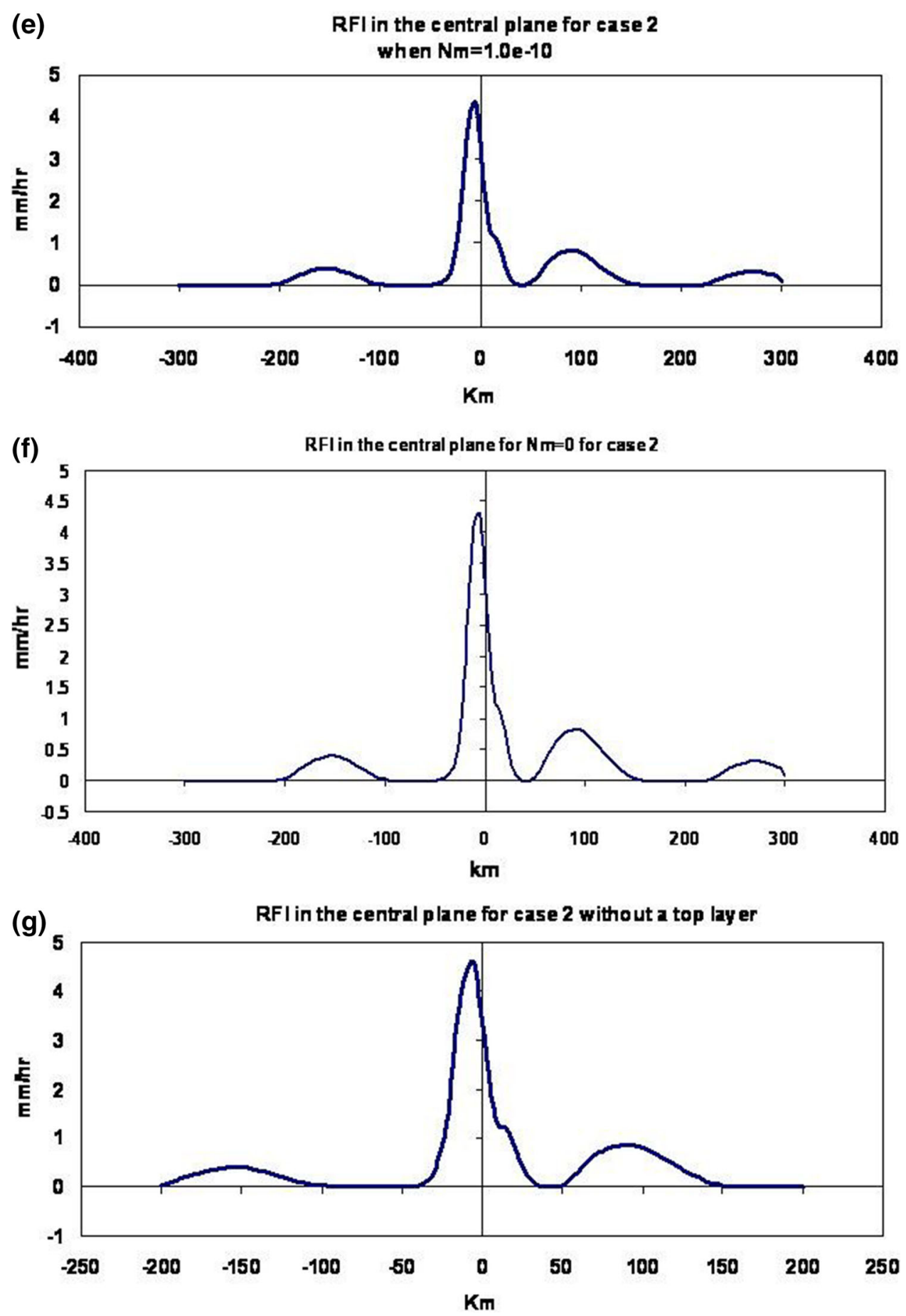

Fig. 3 continued

$8.5 \mathrm{~km}$ and that of top layer has been taken as $4 \mathrm{~km}$ and the above domains have been divided into 34 and 16 discrete layers, respectively, with depth $0.25 \mathrm{~km}$.

The distributions of RFI $(\mathrm{mm} / \mathrm{h})$ in the central plane for strong stable stratification $\left(N_{m}=1.0 e-2,1.0 e-3\right)$, for weak stable stratification $\left(N_{m}=1.0 e-8,1.0 e-10\right)$, for neutral stratification and also for no artificial top layer have been given in figs from $3(\mathrm{~b}-\mathrm{g})$.

From the Fig. 3b-g, it can be seen that pattern in the distributions of RFI $(\mathrm{mm} / \mathrm{h})$ in the central plane are similar whether a top layer is capped or not and also irrespective of top layer stability. From these figs it also appears that if no 

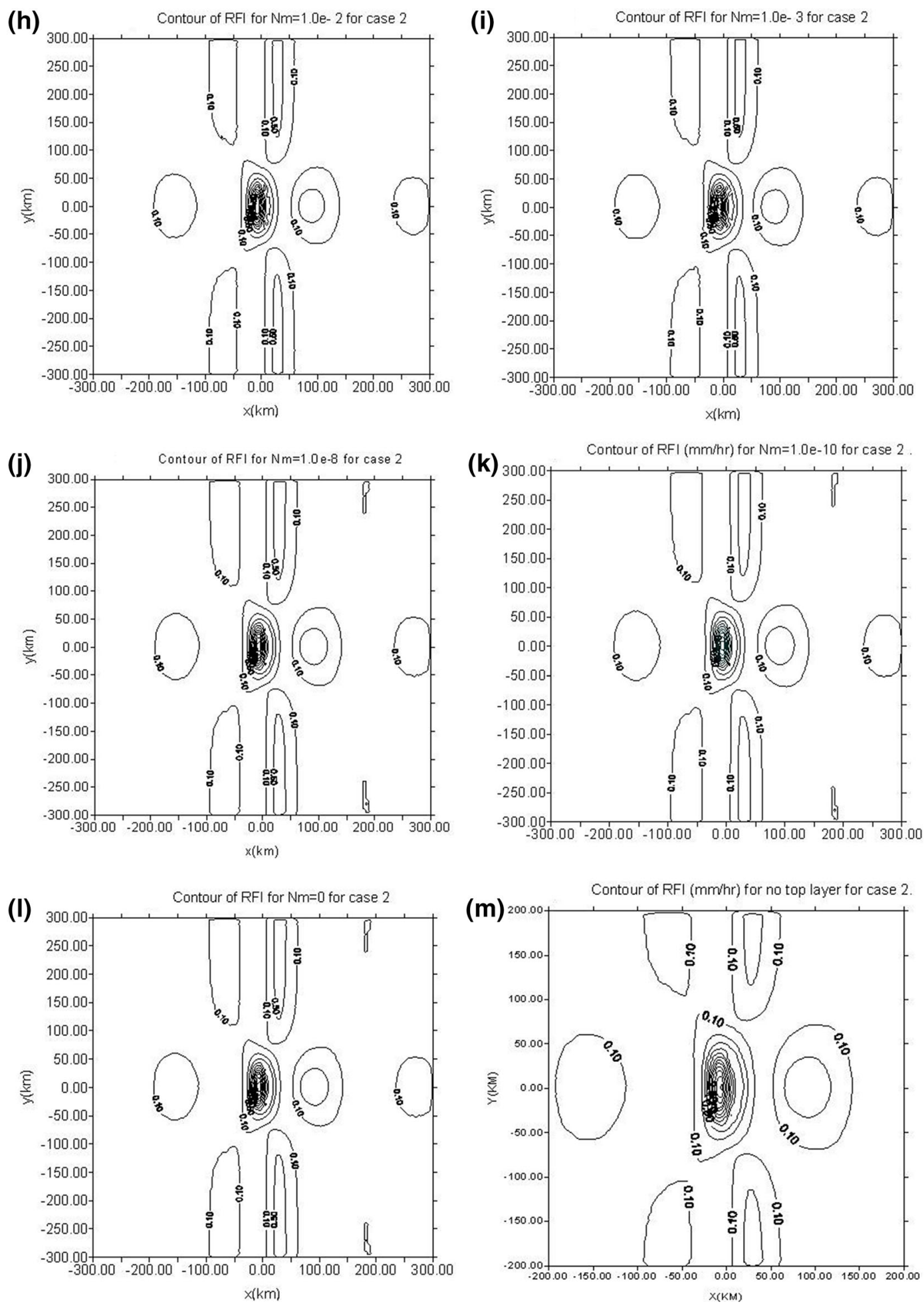

Fig. 3 continued

artificial top layer is capped, then the computed peak RFI is $4.58 \mathrm{~mm} / \mathrm{h}$ located at $5 \mathrm{~km}$ upstream of the peak of the barrier and if an artificial top layer, with any stable/neutral stratification, is imposed then it is about $4.2-4.3 \mathrm{~mm} / \mathrm{h}$ located at the same place and observed RFI was estimated to be $5.0 \mathrm{~mm} \mathrm{~h}^{-1}$ at Lonavla (about $5 \mathrm{~km}$ upstream of the peak). Thus the computed RFI without using any artificial top layer appears to be closer to observed one and imposing 
a top layer is to reduce the magnitude of peak computed RFI and thus underestimation of RFI.Contours of RFI in the 2-dimensional plane with different values of top layer stability are shown in Fig. 3h-m. Pattern of RFI contour is similar to that of case-1. Similar to case-1, here also it can be seen that stability of the top layer has hardly any effect on the spatial (2-D) distribution of computed orographic rainfall.

Reduction in the magnitude of computed maximum RFI may be attributed to the over suppression of the vertically propagating disturbances by the stable artificial top layer.

\section{Conclusion}

Preliminary studies of the cases indicate that

- Given the profile of a 3-D meso-scale mountain, the vertical profile of basic flow across it, the magnitude and spatial distribution (1-D and 2-D) of computed orographic rainfall intensity hardly have any bearing with top layer stability.

- In some cases it has been found that the effect of imposition of a top layer is to reduce the magnitude of peak computed RFI just behind the barrier and thus underestimation of RFI.

- Reduction in the magnitude of computed maximum RFI may be attributed to the over suppression of the vertically propagating disturbances by the stable artificial top layer.

- This result is a 3-D generalization of the earlier 2-D findings of Corby and Sawyar (1958), Palm and Foldvic (1960) and Sawyar (1960).

Acknowledgments Authors express their sincere thanks, on records, to the Director General of Meteorology, India Meteorological
Department, New Delhi, India, for the kind supports provided to carry out this study and to all the staffs and officers of Meteorological training institute for their helps in preparing this paper. I am indebted to my respected teacher \& guide Dr.U.S.De, former Additional Director General of Meteorology, India Meteorological Department, for his kind valuable suggestions.

\section{References}

Collier CG (1975) A representation of the effects of topography on surface rainfall within moving baroclinic disturbances. Q J R Meteorol Soc 101:407-422

Corby GA, Sawyar JS (1958) The airflow over a ridge: the effect of upper boundary condition and high level conditions. Q J R Meteorol Soc 84:25-37

Dutta S (2005) Effect of static stability on the pattern of threedimensional baroclinic lee wave across a meso scale elliptical barrier. Meteorol Atmos Phys 90(3-4):139-152

Dutta S (2007) A meso-scale three-dimensional dynamical model of orographic rainfall. Meteorol Atmos Phys 95(1-2):1-14

Murray R (1948) A note on rainfall of Cherrapunji. Q J R Meteor Soc 74:122-123

Palm E, Foldvic A (1960) Contribution to the theory of two dimensional mountain waves. Geo Publ Oslo 21:1-29

Rhea JO (1978) Orographic precipitation model for hydrometeorological use. Ph.D. thesis, Colorado state university, Atmos Sci Paper No.287, 198

Sarkar RP (1966) A dynamical model of orographic rainfall. Mon Weather Rev 94:555-572

Sarkar RP (1967) Some modifications in a Dynamical model of orographic rainfall. Mon Weather Rev 95:673-684

Sarker RP, Sinha Ray KC, De US (1978) Dynamics of orographic rainfall. Indian J Meteorol Geophys 29:335-348

Sawyar JS (1956) The physical and dynamical problems of orographic rain. Weather 11:375-381

Sawyer JS (1960) Numerical calculation of the displacements of a stratified air stream crossing a ridge of small height. Q J R Meteor Soc 86:326-345

Sinclair MR (1994) A diagnostic model for estimating orographic precipitation. J Appl Meteor 33:1163-1175

Smith RB, Barstad Idar (2004) A linear theory of orographic precipitation. J Atmos Sci 61:1377-1391 